Ann. Biol. anim. Bioch. Biophys., I968, 8 (I), 99-I05.

\title{
HYPOPHYSECTOMIE DE POECILIA RETICULATA (POISSON TÉLÉOSTÉEN)
}

\author{
B. JALABER'T et R. BILLARD \\ avec la collaboration technique de Anne-Marie ESCAFFrE \\ Station centrale de Physiologie animale, \\ Centre national de Recherches zootechniques, 78 - Jouy-en-Josas \\ Institut national de la Recherche agronomique
}

\section{SOMMAIRE}

Une technique d'hypophysectomie par voie operculaire particulièrement simple et rapide, est décrite chez le Guppy Poecilia reticulata. La pénicilline administrée pendant la cicatrisation diminue la mortalité postopératoire.

L'ablation chirurgicale de 1'hypophyse est l'une des techniques essentielles permettant d'aborder l'étude du déterminisme endocrinien de la reproduction. Elle peut également être pratiquée en vue de réaliser un dosage biologique des hormones gonadotropes hypophysaires. Nous rapportons ici une technique simple et rapide, mise au point sur un Poisson Téléostéen vivipare de très petite taille: Poecilia reticulata.

Les opérations habituellement décrites utilisent deux voies d'accès vers l'hypophyse : voie orale ou sub-orale et voie operculaire.

I. Voie orale : Les mâchoires du Poisson sont largement écartées et la commissure labiale parfois sectionnée et élargie. L'os parasphénoïde est dégagé par une incision des tissus du palais, puis entamé à l'aide d'une fraise à main ou d'une pince fine, de manière à dégager une fenêtre par laquelle l'hypophyse sera aspirée à l'aide d'une pipette (Vrvikn, I94I, sur Gobius paganellus et sur Scylliorhinus ; TAvol,Ga, I955, sur Bathygobius soporator; ChAmbolle, I964, sur Gambusia; Donar.DSon et MCBRIDE, I967 sur Salmo Gairdnerii). MathEws, (r933), utilise la voie sub-orale chez Fundulus en pratiquant une incision en $\mathrm{V}$ à travers le plancher buccal, ce qui a pour inconvénient de sectionner un vaisseau avec risque d'hémorragie.

2. Voie operculaire: ABramowitz, (I937), sur Fundulus, écarte l'opercule droit et atteint 1 'hypophyse après avoir écarté les $\mathrm{I}^{\mathrm{er}}$ et $2^{\mathrm{e}}$ ares branchiaux. Plusieurs techniques utilisant cette voie ont été décrites avec des variantes imposées par les différences antomiques entre espèces (ChAvIN, I956, sur Carassius auratus; BARR, I963 a, sur la Plie (Pleuronectes platesa); HANDIN, I964, sur Tilapia Mossambica; AHSAN, I964, sur Couesius plumbeus) ; BELSARE, I965, sur Ophicephalus 
punctatus; Ball, I965 sur Poecilia latipinna) ; SundararaJ et Goswamr, I965, utilisent également la voie operculaire sur Heteropneustes fossilis, mais ils éclairent directement la cavité buccale en introduisant une sourcelumineuse froide parla bouche.

Beaucoup de ces techniques, telles qu'elles furent décrites, sont en général traumatisantes, et d'autant plus préjudiciables à la survie ultérieure que 1'animal est plus petit et fragile. Sur Poecilia reticulata, nous avons réussi à éviter toute incision operculaire, en limitant 1'opération à l'incision transversale de l'os parasphénoïde et des tissus épithéliaux qui le recouvrent. Les difficultés résultent essentiellement de la petite taille des animaux opérés : 20 à $30 \mathrm{~mm}$ pour les mâles et 25 à $45 \mathrm{~mm}$ pour les femelles.

\section{MATÉRIEL, ET TECHNIQUE OPÉRATOIRE,}

L'opération est réalisée sous loupe binoculaire à éclairage axial et commande de mise au point au pied. Le Poisson est placé sur le dos dans une gouttière creusée dans de la pâte à modeler, légèrement incliné sur le flanc droit, la tête en contre-bas, l'axe du corps faisant un angle de $15^{\circ}$ avec l'horizontale. L'opercule gauche est écarté à l'aide d'un premier micro-écarteur ; les branchies sont rabattues du côté opposé et maintenues en place par l'autre écarteur (fig. I). Le fond de la cavité

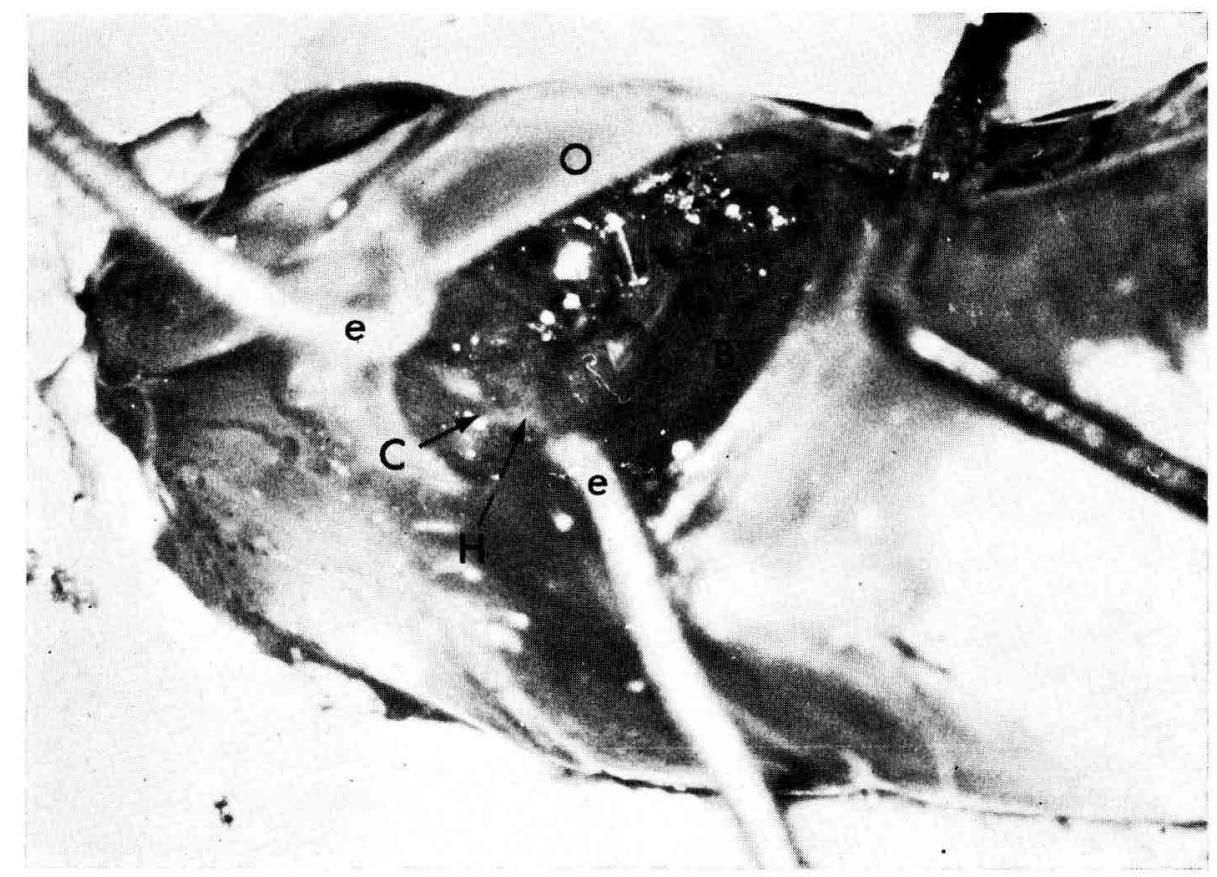

FIG. I. - Position de l'animal pour opération

(L'hypophyse est visible par transparence, à travers l'os parasphénoïde)

$\mathrm{H}$ : Hypophyse ; $\mathrm{C}$ : Chiasma optique ; $\mathrm{O}$ : Opercule ;

$\mathrm{B}$ : Branchies; $e$ : écarteur.

ainsi dégagé, montre l'hypophyse en arrière des nerfs optiques, visible par transparence à travers l'os parasphénoïde qui fait saillie à cet endroit précis. Les tissus du palais sont alors incisés avec un fragment de lame de rasoir monté, dégageant le parasphénoïde, qui est à son tour sectionné transversalement, au-dessus de la partie antérieure de l'hypophyse, en arrière du foramen de l'efférente pseudobranchiale. 
A l'aide d'une micropipette d'extrémité recourbée, d'un diamètre de 200 à $250 \mu$ pour la femelle et de 100 à $150 \mu$ pour le mâle, on fait alors pression sur la partie antérieure du parasphénoïde qui s'effondre partiellement. L'hypophyse est très légèrement repoussée en partie postérieure, et apparaît dans l'espace créé entre les deux bords de la section; on peut alors l'aspirer dans la pipette (fig. 2). Par la suite, la partie antérieure de l'os parasphénoïde revient en place d'elle-même, avec parfois un léger chevauchement des deux parties, comme le montrent les examens histologiques de contrôle.

Lorsque l'opération est bien menée il n'est pas nécessaire d'élargir la commissure operculaire, ni d'effectuer aucune autre section que celle du plancher crânien. L'artère efférente pseudobranchiale peut parfois être lésée, mais le sang coagule très vite, et la survie ultérieure de l'animal n'en paraît pas

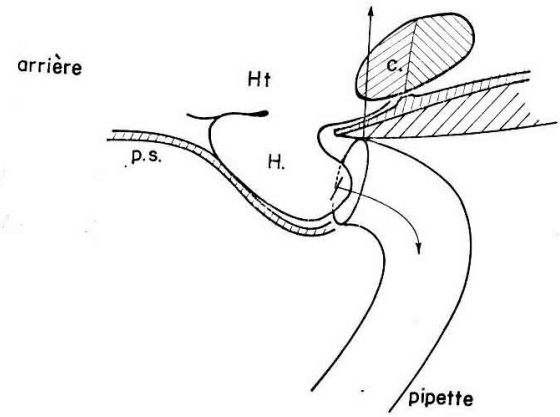

Fig. 2. - Mécanisme de l'opération

Ht : hypothalamus; $\mathrm{H}$ : hypophyse ; p.s. : parasphénoïdes; C : chiasma optique. affectée.

Un opérateur entraîné peut réaliser I 2 à 20 hypophysectomies à l'heure.

\section{CONTRÔLE DE L'HYPOPHYSECTOMIE}

Pour vérifier le succès de l'opération, nous avons contrôlé, d'une part l'intégrité de 1'hypophyse par observation immédiate sous microscope après prélèvement (fig. 3),

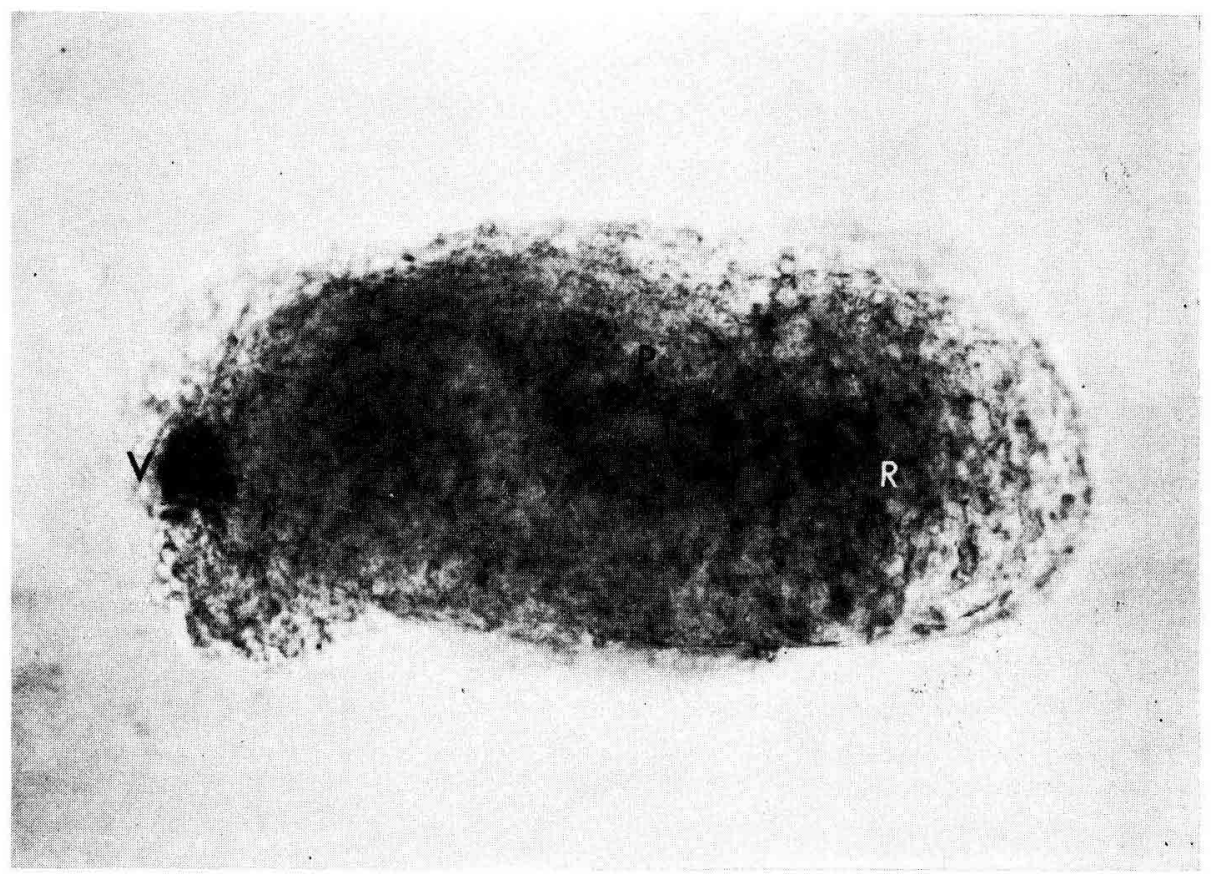

Fig. 3. - Hypophyse après prélèvement à la pipette

V : Veine hypophysaire $\mathrm{R}$ : Pars distalis rostrale ; P : Pars distalis proximale ; I : Pars intermedia 


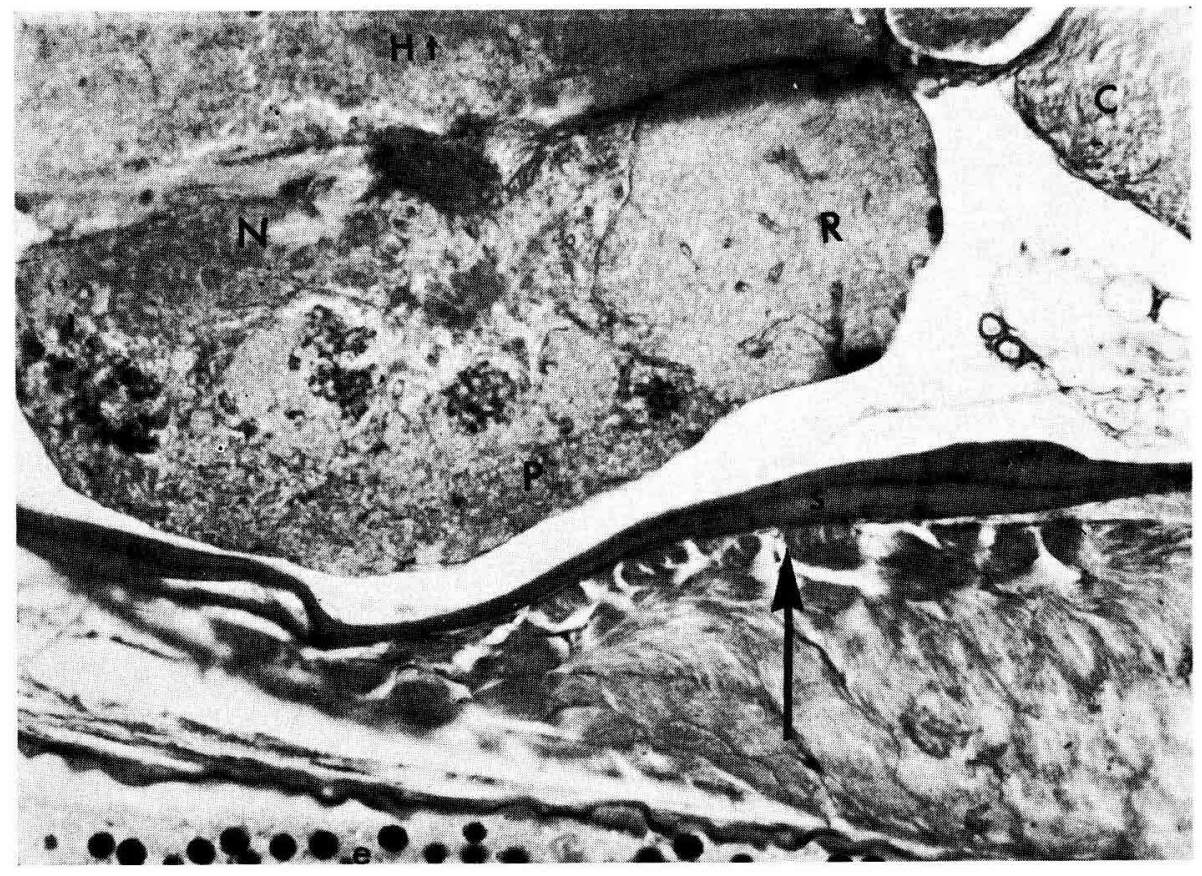

FIG. 4 a. - Contrôle hislologique de l'hypophysectomie Animal intact ;

Ht : Hypothalamus ; $s$ : Parasphénoïde ; R : Pars distalis rostrale ; $\mathrm{P}$ : Pars distalis proximale; I,: Pars intermédia; $\mathrm{N}$ : Neurohypophyse ; $e$ : Épithélium buccal ;

La flèche indique l'endroit de la section de l'os parasphénoïde

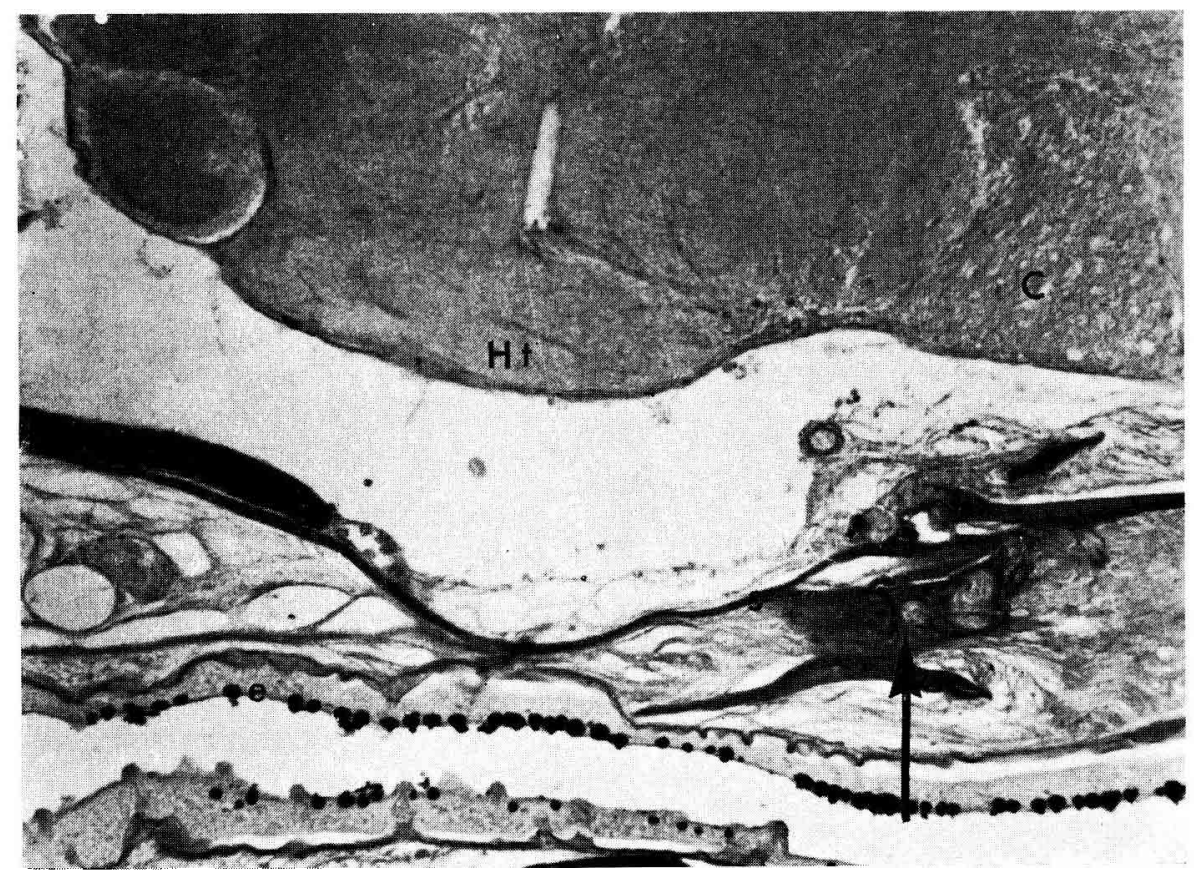

FIG. 4 b. - Cortrôle histologique de l'hypophysectomie Animal hypophysectomisé 
d'autre part l'absence de fragments hypophysaires sur coupes histologiques (fig. $4 a$ et $4 b)$.

La confrontation des observations nous a montré que la vérification directe immédiate suffit. Seule l'ablation de la neuro-hypophyse peut être plus ou moins complète suivant les cas.

\section{SURVIE DES ANIMAUX HYPOPHYSECTOMISÉS}

Il est maintenant bien connu que 1'hypophysectomie réduit considérablement l'aptitude d'un certain nombre de Téléostéens euryhalins à survivre en eau douce.

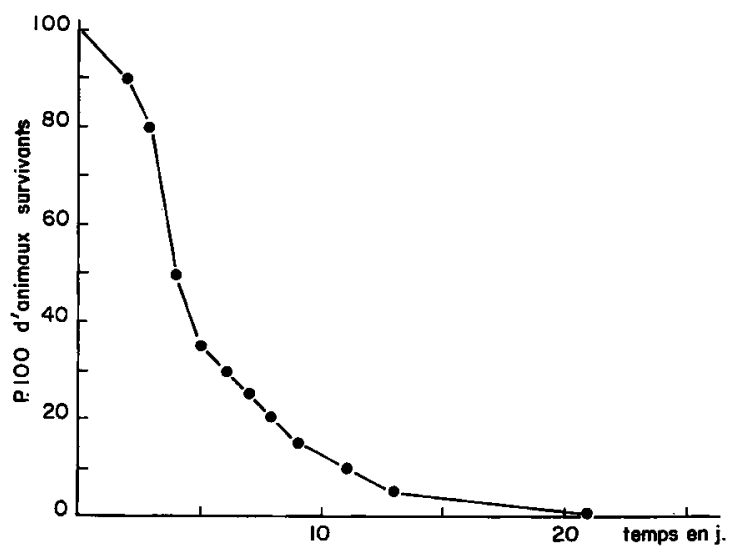

FIG. 5. - Courbe de survie des animaux hypophysectomisés placés en eau douce (milieu avec pénicilline) - (20 mâles)

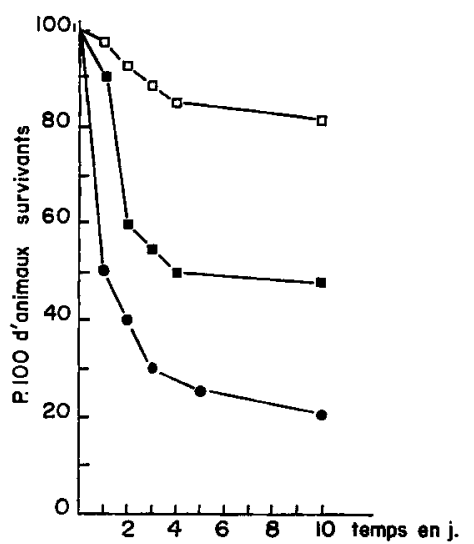

Fig. 6. - Effet de différents traitements d la pénicilline, sur la survie d court terme des femelles hypophysectomisées

-- Aucun traitement (Io animaux).

- Traitement pendant 1 jour après opération, à raison de 200000 UI/litre ( 25 animaux).

- - Traitement de quelques heures avant opération et pendant 4 jours après opération, à raison de 200000 UI/litre (25 animaux).

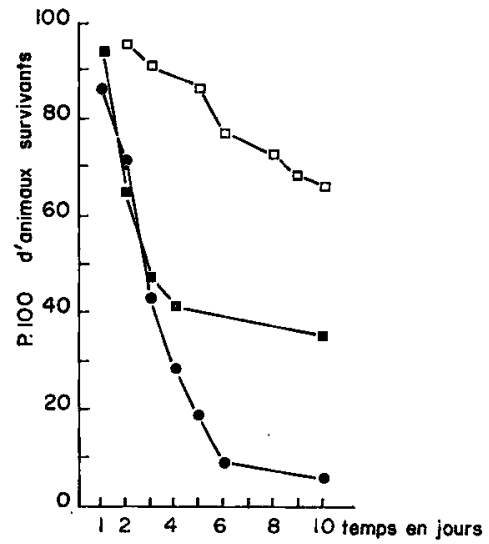

FIG. 7. - Effets de différents traitements a to pénicilline, sur la survie d court terme des mâles hypophyseclomisés

-- Aucun traitement (2I animaux).

- Traitement pendant I jour après opération, à raison de $200000 \mathrm{UI} /$ litre (i 7 animaux).

- - - Traitement de quelques heures avant opération et pendant 4 jours après opération. à raison de 200000 UÍ/litre (22 animaux). 
Poecilia reticulata peut s'adapter et vivre en eau salée, mais vit et se reproduit normalement en eau douce. Or, après hypophysectomie, la survie de nos animaux en eau douce n'a jamais excédé ro jours (fig. 5). Pour cette raison, les Poissons opérés ont été placés dans une solution équilibrant au mieux la pression osmotique sanguine : $\mathrm{NaCl}$ à $8 \mathrm{p}$. roo ou bien eau de mer reconstituée diluée au tiers.

Du fait des difficultés présentées par une amélioration des conditions d'aseptie de l'opération et du milieu post-opératoire, nous avons essayé avec succès différentes doses de pénicilline dans le milieu pré et post-opératoire (fig. 6, 7).

I. Le traitement à la pénicilline pendant I à 4 jours, améliore significativement la survie post-opératoire (200 $000 \mathrm{UI} / 1$ de pénicilline $\mathrm{G}$ pour les femelles).

2. Des doses trop élevées de pénicilline administrées pendant toute la durée de la cicatrisation (200 $000 \mathrm{UI} / 1$ durant 4 jours avec renouvellement quotidien) semblent préjudiciables à la survie à long terme. La mortalité des Témoins non opérés placés dans les mêmes conditions est même supérieure au bout de 30 jours à celle des hypophysectomisés. Il est possible que la pénicilline à hautes doses perturbe le métabolisme et particulièrement celui des témoins d'un niveau plus élevé que celui des animaux privés d'hypophyse (fig. 8).

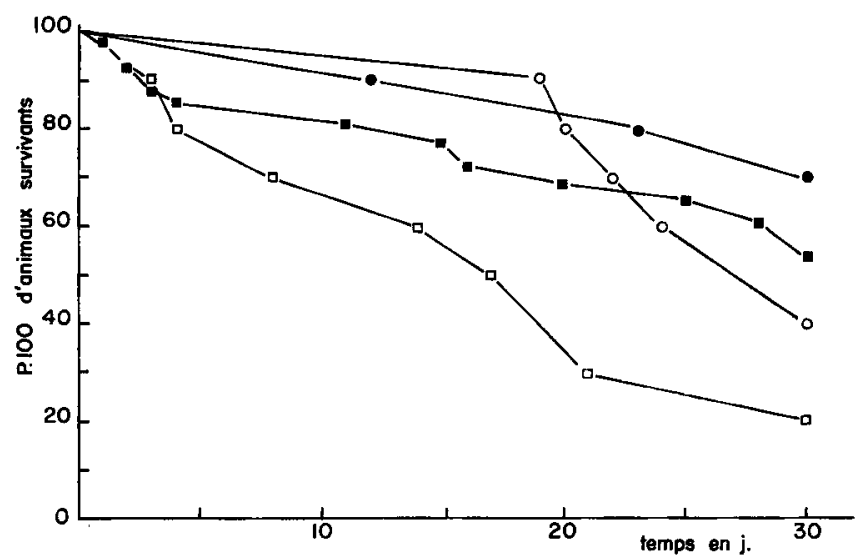

FIG. 8. - Effet néfaste à long terme, de trop fortes doses de pénicilline.

- -9 hypophysectomisées, traitées 4 jours à 200000 UI/litre (25 animaux).

- + - non opérées, même traitement (Io animaux).

- - o hypophysectomisées, traitées I jour à 200000 UI/litre, puis 3 jours à r 00000 UI/litre (ro animaux).

$-0-$ q non opérées, même traitement (Io animaux).

Excepté un essai en eau douce, toutes ces expériences de survie ont été réalisées en eau de mer reconstituée, diluée au tiers.

C'est pourquoi le protocole que nous avons maintenant adopté utilise des doses plus faibles et nous donne satisfaction.

- Pour les femelles : pré-traitement de quelques heures à $200000 \mathrm{UI} / 1$; traitement post-opératoire de I jour à 200 ooo UI/1 puis de 3 jours à roo ooo UI/1.

- Pour les mâles : nous avons été conduits à utiliser des doses de pénicilline deux fois moindres, du fait d'une sensibilité apparemment encore plus grande aux effets à long terme. 
La technique d'hypophysectomie pratiquée par voie operculaire sur Poecilia reticulata est rapide et peut permettre grâce à une survie satisfaisante au bout de 30 jours, la constitution de lots expérimentaux importants, en vue d'un étude analytique des effets de diverses supplémentations hormonales.

Reçu pour publication en décembre 1967.

\section{SUMMARY}

HYPOPHYSECTOMY IN THE GUPPY " POECILIA RETICULATA "

A quick easy surgical technique of hypophysectomy using opercular approach is described in the Guppy Poecilia reticulata. The addition of penicillin to the water during the healing phase reduces poset-operative lethality.

\section{RÉFÉRENCES BIBLIOGRAPHIQUES}

Abramowitz A. A., 1937. The opercular approach to the pituitary. Science, 85, 609.

AshaN N., I964. The control of cyclical changes in the testicular activity of the lake chub Couesius Plumbeus. (Agassiz). Thèse Univ. Colombie Britannique.

Ball J. N., 1965. Partial hypophysectomy in the Teleost Poecilia; Separate identities of Teleostean growth hormone and Teleostean prolactin-like hormone. Gen. Comp. Endocr., 5, 654-66r.

BARR W. A., I963: The endocrine control of the sexual cycle in the plaice Pleuronectes platessa L. I. Cyclical changes in the normal ovary. Gen. Comp. Endocr., 3, 197-225.

Belsare D. K., 1965. Changes in gonads and the thyroid gland after hypophysectomy in Ophiocephalus punclatus Bloch. J. Exp. Zool., 158, $\mathrm{x}-8$.

Chambolle P., I964. Influence de l'hypophysectomie sur la gestation de Gambusia sp. (Poisson Téléostéen). C. R. Acad. Sci. Paris, 259, 3855-3857.

Chavin W., 1956 a. Pituitary-adrenal control of melanization in Xanthic goldfish, Carassius auratus. J. Exp. Zool., 133, I-36.

Donaldson E. M., McBride J. R., 1967. The effects of hypophysectomy in the rainbow trout Salmo Gairdnerii with special reference to the pituitary-Interrenal axis. Gen. Comp. Endocrinol, 9, 93-IoI.

Fontaine M., Callanand O., Olivereau M., 1949. Hypophyse et Euryhalinité chez l'Anguille. $C . R$. Acad. Sci., Paris, 228, 513-514.

HANDiN R. I., et al., I964. The effect of hypophysectomy on survival and on the thyroid and interrenal histology of the cichlid Teleost Tilapia mossanbica. J. Exp. Zool., 157, 339-344.

Matrhews S. A., 1939. The relationship between the pituitary gland and the gonads in Fundulus. Biol. Bull., 76, 240-255.

Olivereau M., Ball J. N., I964. Contribution à l'histophysiologie de l'hypophyse des Téléostéens en particulier de celle de Poecilia sp. Gen. Comp. Endocr., 4, 523-532.

Pickford G. E., 1953. A study of the hypophysectomized male killifish Fundulus heteroclitus (L.). Bull. Bingham. Ocean Coll., 14, 5-4I. (Cité par J. N. BaLl I965).

Sundararaj B. I., Goswami S. V., 1965 . "Seminal vesicle "Response of intact, castrate, and hypophysectomized Goldfish, Heteropneustes fossilis (BLOCH) to testosterone propioniate, prolactin and growth hormone. Gen. Comp. Endocr., 5, 464-474.

TAvolGa W. N., 1.955. Effects of gonadectomy and hypophysectomy on prespawning behaviour in males of the Gobiid fish Bathygobius Soporator. Physiol. Zool., 28, 218-233.

VIvien J. H., I94I. Contribution à l'étude de la physiologie hypophysaire dans ses relations avec l'appareil génital, la thyroìde et les corps suprarénaux chez les Poissons Sélaciens et Téléostéens, Scylliorhinus canicula et Gobius paganellus. Bull. Biol. France et Belgique, 75, 257-309. 